\title{
基于拉矫过程参数组合优化的带钢酸洗效率*
}

\author{
张赵宁 张杰 孔宁 李洪波 \\ (北京科技大学机械工程学院 北京 100083)
}

\begin{abstract}
摘要: 为研究拉矫过程中张力与弯矩组合条件对热轧带钢酸洗效率的影响, 以热轧电工钢板为例, 采用弹塑性力学分析方法 讨论张力、弯矩对曲率和伸长率的影响, 建立带钢在拉弯矫直过程中, 曲率、伸长率随张力/弯矩变化的数学模型。以曲率、 伸长率为目标值, 通过不同塑性弯曲状态下的静力平衡方程组, 构建不同目标值下张力和弯矩的关系。采用电化学酸洗试验, 分别研究不同曲率和伸长率对酸洗时间的影响, 建立酸洗时间缩短率的预测模型。以酸洗时间缩短率为评价标准, 分别研究 不同曲率和伸长率对酸洗效率的影响, 并计算出带钢在达到酸洗饱和点时的相对曲率和伸长率分别为 2.33 和 $1.51 \%$ 。以不同 目标曲率、伸长率时的张力-弯矩曲线作为左右边界, 通过小张力-大弯矩条件下的相对张力临界值确定上下边界, 计算出张 力-弯矩调节域, 研究结果可为酸洗前拉弯矫直过程参数组合优化提供理论依据。
\end{abstract}

关键词: 拉弯矫直; 曲率; 伸长率; 酸洗; 张力-弯矩调节域

中图分类号: TG142

\section{Strip Pickling Efficiency on the Basis of Combined Parameters Optimization during Tension Leveling Process}

\author{
ZHANG Zhaoning ZHANG Jie KONG Ning LI Hongbo \\ (School of Mechanical Engineering, University of Science and Technology Beijing, Beijing 100083)
}

\begin{abstract}
In order to study the combined effect of tension and bending moments conditions on the acid pickling process of hot rolled strips, an elastic-plastic mechanics analysis method is conducted to analyze the influence of tension and bending moment on the curvature and elongation. Taking hot-rolled electrical steel sheet as an example, a mathematical model is established on the consideration of the relationship between curvature/elongation and tension/bending moment. Regarding the target values of curvatures and elongations, the relationship between tension and bending moment is established through the static equilibrium equations under various plastic bending stages. Electrochemical pickling experiments is also applied to investigate the effects of different curvatures and elongations on the acid pickling time, and to build a prediction model about the pickling time shortening rate with curvature/elongation. On the basis of pickling time shortening rate, the pickling efficiency is assessed with different curvatures and elongations. The saturation points of curvature and elongation during the process of pickling are 2.33 and $1.51 \%$, respectively. The tension-bending moment regulatory domain is obtained, which treats the tension-bending moment relation curves as the left and right boundaries with different curvature and elongation values. The upper and lower boundaries are determined by the critical tension values under small tension and large bending moment conditions. The proposed regulatory domain provides a theoretical guidance for the configuration optimization of a tension leveling before the acid pickling process of hot rolled strips.
\end{abstract}

Key words: tension leveling; curvature; elongation; acid pickling; tension-bending moment regulatory domain

\section{0 前言}

拉弯矫直机作为冷轧生产必不可少的设备, 广 泛应用于酸洗、镀锌、连退等工序的入口处, 其在 改善板形缺陷、机械除鳞等方面发挥了重要作用 ${ }^{[1]}$ 。

* 国家自然科学基金(51605026)和中央高校基本科研业务费专项资金 (FRF-TP-15-082A1)资助项目。20180319 收到初稿, 20180718 收到 修改稿
当前针对带钢拉弯矫直的研究, 主要侧重于内应力 的释放、板形缺陷的改善等方面 ${ }^{[2-7]}$, 并且已经取得 显著的效果。酸洗生产线的下游通常跟随有冷轧、 平整等进一步改善板形的工序 ${ }^{[8]}$, 因此针对酸洗入 口处拉弯矫直机的研究, 通常更注重于如何充分发 挥拉矫破鳞功能、提高带钢表面质量、缩短带钢酸 洗时间等方面。

拉弯矫直过程中, 氧化铁皮的破碎程度取决于 拉弯矫直机张力-弯矩的组合条件。目前针对拉弯矫 
直对氧化铁皮剥离性能的研究工作主要集中于对比 拉伸和弯曲效果的优劣。叶东东等 ${ }^{[9-11]}$ 对热轧带钢 进行不同应力状态的破鳞试验, 发现在压应力作用 下的氧化铁皮剥落量要大于拉应力作用下的氧化铁 皮剥落量。陈兵等 ${ }^{[12]}$ 通过有限元仿真计算发现, 在 拉矫机的总张力和总插入深度不变的前提下, 当后 弯曲辊的插入深度值大于前弯曲辊插入深度时可获 得更好的破鳞效果。杨子良等 ${ }^{[13]}$ 利用自主研发的拉 矫破鳞试验机对热轧带钢进行了拉矫模拟试验, 发 现拉弯矫直过程中带钢的伸长率主要由破鳞辊的压 下量决定。付松岳等 ${ }^{[14]}$ 对比了拉伸法、划痕法、弯 曲法的破鳞效果, 发现在弯曲作用下氧化铁皮的剥 落量最大。

EVANS 等 ${ }^{[15-17]}$ 研究发现, 带钢在拉弯矫直过程 中, 上下表面的不同变形过程导致氧化铁皮出现不 同的破裂方式。带钢与弯曲辊接触的下表面在弯曲 作用下产生压缩塑性变形, 此时下表面受到的压应 力随着带钢中心层曲率的增加逐渐增大, 中心层弯 曲曲率的大小间接地决定了下表面氧化铁皮的破裂 程度。王斌等 ${ }^{[18]}$ 通过纳米压痕和拉伸试验的方法测 得氧化铁皮的弹性模量及氧化铁皮-基体的结合力 系数, 并建立了薄板氧化铁皮的有限元模型。其研 究表明在压应力作用下, 当氧化铁皮剥落面积达到 $24.1 \%$ 时会出现饱和。而带钢的另一面在拉弯矫直 过程中受张力拉伸作用产生塑性延伸, LI 等 ${ }^{[19]}$ 研究 发现氧化铁皮的剥落量随着拉弯矫直机伸长率设定 值的增大而增多, 且带钢的伸长率在提高的过程中 存在饱和点。王尚等 ${ }^{[20]}$ 采用拉伸试验研究了 Q235-A 在拉伸作用下的开裂行为, 发现试样氧化铁皮的裂 纹条数随着拉伸应变增加呈现慢、快、慢的变化规 律, 当应变为 $0.05 \%$ 时, 氧化铁皮表面开始产生裂 纹, 当应变大于 $0.15 \%$ 时, 裂纹条数几乎不再随着 应变的增加而增多。

拉矫破鳞的最终目的是为了缩短酸洗时间。相 对于拉矫过程中氧化铁皮的剥落量, 酸洗时间能更 直观地反映破鳞效果, 且更准确地确定拉伸、弯曲 过程中的饱和点。拉弯矫直过程中酸洗时间取决于 弯曲曲率和伸长率的大小, 而弯曲曲率和伸长率又 取决于张力-弯矩的组合条件。为了研究不同的张 力、弯矩组合条件对酸洗时间的影响, 本文采用弹 塑性力学分析方法, 建立带钢在拉弯矫直过程中曲 率、伸长率随张力-弯矩变化的数学模型。定义曲率、 伸长率为目标变量, 构建不同目标值下张力-弯矩的 关系曲线。通过电化学酸洗试验, 建立酸洗时间缩 短率预测模型, 计算曲率和伸长率酸洗饱和点。以 弯曲曲率、伸长率为中间变量, 分析拉弯矫直参数
对酸洗时间的影响, 并计算张力-弯矩调节域, 为酸 洗前拉弯矫直过程参数组合优化提供理论依据。

\section{1 拉弯矫直模型}

由当前的研究 ${ }^{[18-19]}$ 可知, 拉弯矫直机的破鳞效 果取决于弯曲曲率和伸长率的大小, 而弯曲曲率和 伸长率取决于拉弯矫直机张力-弯矩的组合条件。为 了研究带钢在拉弯矫直过程中, 张力-弯矩组合条件 对带钢酸洗效率的影响, 首先需要分析拉弯矫直过 程中, 张力-弯矩对弯曲曲率和伸长率的影响。采用 弹塑性力学分析方法, 建立拉弯矫直数学模型, 并 构建拉弯矫直过程中, 曲率、伸长率与张力-弯矩的 关系。图 1 为拉弯矫直机矫直单元的工作示意图, $L_{0}$ 为辊间距, $\delta$ 为弯曲辊插入深度, $h$ 为带钢厚度, $M$ 为弯矩, $T_{1} 、 T_{2}$ 为前后张力。由图 1 可知, 带钢 在拉弯矫直时的弯矩图与集中载荷作用下的简支梁 弯矩图相似。因此在不考虑带钢纵向纤维条之间正 应力影响的情况下, 可以将拉弯矫直的单次弯曲过 程简化成两端有轴向力作用、中间受集中载荷作用 的简支梁模型。

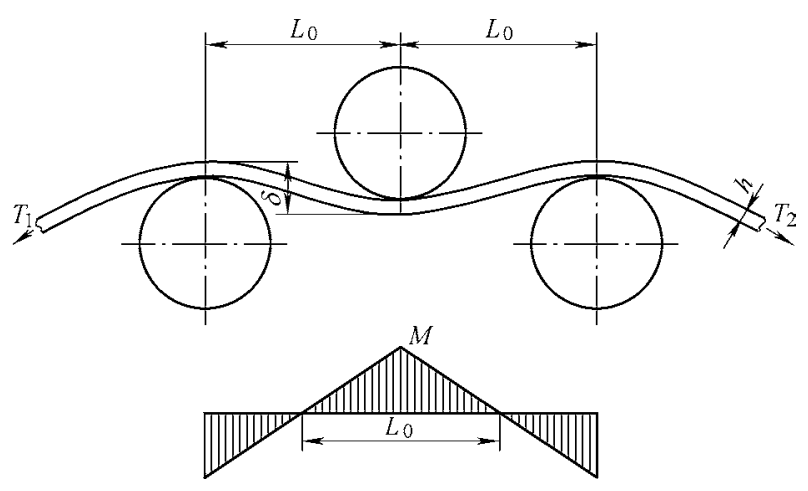

图 1 拉弯矫直机矫直单元的工作示意图

\section{1 条件与假设}

为建立带钢拉弯矫直模型, 作如下基本假设。

(1) 纵向纤维条间无正应力假设: 将带钢沿宽 度方向分成若干个纵向纤维条, 不考虑纵向纤维条 之间的相互影响。

(2) 平面假设: 纵向纤维条变形后, 其横截面 仍保持平面, 并垂直于变形后纵向纤维条的中心层。

（3）线弹性假设: 带钢厚度方向的应变与其跟 中性层的距离成正比, 且在弹性区间内遵循胡克 定律。

（4）线性强化假设 ${ }^{[21]}$ : 为了使模型计算结果接 近于实际条件, 假设带钢为线性强化弹塑性体, 线 性强化系数为 $\mu=E_{\mathrm{p}} / E$, 其中 $E$ 为带钢的弹性模 量, $E_{\mathrm{P}}$ 为带钢的加工硬化模量。 
(5) 集中载荷假设: 忽略弯曲辊半径对弯曲变 形的影响, 假设弯曲辊的压入点为集中载荷。

(6) 张力相等假设: 假设带钢在拉弯矫直过程 中, 前后张力大小相等、方向相反。

(7) 忽略包辛格效应。简化后的模型如图 2 所 示。由图 2 可知, 简化后的模型基本参数: 跨距为 $L_{0}$, 轧制力为 $F$, 弯矩为 $M=F L_{0} / 4$, 张力为 $T$ 。

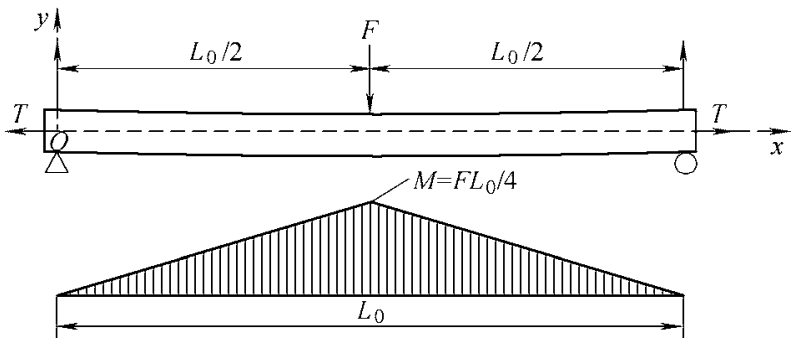

图 2 简化后带钢的拉矫模型示意图

\section{2 曲率、伸长率与应力分布特征参数的关系}

若带钢的屈服应力为 $\sigma_{\mathrm{s}}$, 则带钢在弹性极限状 态下的弯矩 $M_{\mathrm{e}}$ 、张力 $T_{\mathrm{e}}$ 、曲率 $\kappa_{\mathrm{e}}$ 分别为

$$
\begin{gathered}
M_{\mathrm{e}}=\frac{\sigma_{\mathrm{s}} b h^{2}}{6} \\
T_{\mathrm{e}}=\sigma_{\mathrm{s}} b h \\
\kappa_{\mathrm{e}}=\frac{1}{\rho_{\mathrm{e}}}=\frac{2 \sigma_{\mathrm{s}}}{E h}=\frac{2 \varepsilon_{\mathrm{s}}}{h}
\end{gathered}
$$

式中, $h$ 为带钢厚度, $b$ 为简化后带钢的宽度, $E$ 为 弹性模量, $\varepsilon_{\mathrm{s}}$ 为屈服应变, $\rho_{\mathrm{e}}$ 为弹性极限时带钢的 曲率半径。

基于塑性弯曲理论知识 ${ }^{[22]}$, 可分别定义量纲一 参数

$$
m=\frac{M}{M_{\mathrm{e}}} \quad t=\frac{T}{T_{\mathrm{e}}} \quad k=\frac{\kappa}{\kappa_{\mathrm{e}}}=\frac{1 / \rho}{1 / \rho_{\mathrm{e}}}
$$

式中, $M 、 T 、 \rho 、 \kappa$ 分别表示带钢的真实弯矩、真 实张力、中性层的真实曲率半径和中性层的真实曲 率。 $m 、 t 、 k$ 分别表示相对弯矩、相对张力、中性 层的相对曲率。

带钢在拉伸弯曲的过程中, 断面的纤维层随张 力和弯矩的不同条件而表现出不同的应力应变状 态: (1) 纯弹性弯曲阶段(图 3a); (2) 单侧塑性弯曲 阶段(图 3b); (3) 双侧塑性弯曲阶段(图 3c)。其中上 表面为受拉侧, 下表面为受压侧。

带钢在拉伸弯曲时, 中性层发生偏移, 其偏心 距为 $d$ 、弹性区高度为 $c$, 定义量纲一参数

$$
\eta=d /\left(\frac{h}{2}\right) \quad \gamma=c /\left(\frac{h}{2}\right)
$$

式中, $\eta$ 表示中心层的相对偏心距, $\gamma$ 表示弹性区的 相对高度。

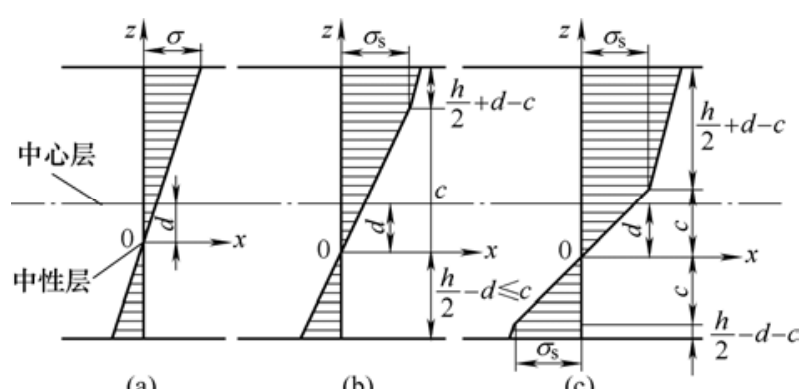

(a)

(b)

(c)

图 3 拉伸弯曲不同状态时的应力分布图

在塑性弯曲阶段的弹性区内, $z=c$ 处的应力为 $\sigma_{\mathrm{s}}$ 。根据假设(3)可知

$$
\varepsilon_{\mathrm{s}}=\frac{C}{\rho}=\frac{\sigma_{\mathrm{s}}}{E}
$$

根据式(6)可知, 弹塑性阶段中性层的曲率半 径、相对曲率分别为

$$
\begin{gathered}
\frac{1}{\rho}=\frac{\sigma_{\mathrm{s}}}{E C}=\frac{2 \sigma_{\mathrm{s}}}{E h \gamma}=\frac{1}{\rho_{\mathrm{e}}} * \frac{1}{\gamma} \\
k=\frac{1 / \rho}{1 / \rho_{\mathrm{e}}}=\frac{1}{\gamma}
\end{gathered}
$$

由于带钢在拉伸弯曲过程中, 中性层相对于中 心层的偏心距为 $d$, 则中心层的曲率半径 $\rho_{\mathrm{c}}$ 和相对 曲率 $k_{\mathrm{c}}$ 分别如式(9)、(10)所示

$$
\begin{gathered}
\rho_{\mathrm{c}}=\rho+d=\rho+\frac{h \eta}{2} \\
k_{\mathrm{c}}=\frac{1 / \rho_{\mathrm{c}}}{1 / \rho_{\mathrm{e}}}=\frac{\rho_{\mathrm{e}}}{\rho+\frac{h \eta}{2}}=\frac{k}{1+\varepsilon_{\mathrm{s}} \eta k}
\end{gathered}
$$

带钢在拉伸弯曲时, 上表面为受拉侧。根据假 设(3)可知上表面的伸长率 $\varepsilon_{\mathrm{a}}$ 为

$$
\varepsilon_{\mathrm{a}}=\frac{z}{\rho}=\frac{1}{\rho}\left(\frac{h}{2}+d\right)=k \varepsilon_{\mathrm{s}}(1+\eta)
$$

将式(8)分别代入式(10)、(11)可得到拉伸弯曲的 塑性弯曲阶段, 中心层相对曲率和上表面伸长率的 表达式

$$
\begin{gathered}
k_{\mathrm{c}}=\frac{k}{1+\varepsilon_{\mathrm{s}} \eta k}=\frac{1}{\gamma+\varepsilon_{\mathrm{s}} \eta} \\
\varepsilon_{\mathrm{a}}=k \varepsilon_{\mathrm{s}}(1+\eta)=\frac{\varepsilon_{\mathrm{s}}(1+\eta)}{\gamma}
\end{gathered}
$$

\section{3 张力、弯矩与曲率和伸长率的关系}

根据带钢在拉伸弯曲的纯弹性弯曲阶段应力分 布图(图 3a)可知, 弹性阶段上表面的应力方程为

$$
\sigma=\frac{E\left(d+\frac{h}{2}\right)}{\rho}=\sigma_{\mathrm{s}} k(\eta+1)
$$

根据纯弹性阶段的力矩平衡关系和轴向力平衡 
关系有

$$
\begin{gathered}
m=\frac{M}{M_{\mathrm{e}}}=\frac{1 / \rho}{1 / \rho_{\mathrm{e}}}=k \\
t=\eta \frac{1 / \rho}{1 / \rho_{\mathrm{e}}}=\eta k
\end{gathered}
$$

将式(15)、(16)代入式(14)可将弹性弯曲阶段上 表面的应力方程化简为式(17)所示

$$
\sigma=\sigma_{\mathrm{s}}(m+t)
$$

当带钢的上表面的应力达到弹性极限 $\sigma_{\mathrm{s}}$ 时, 带 钢由纯弹性阶段进入单侧塑性阶段, 此时的边界条 件称为第一边界条件。根据式(17)可知第一边界条 件为

$$
m+t=1
$$

\subsection{1 单侧塑性弯曲阶段}

单侧塑性弯曲阶段时断面的应力分布如图 3b 所示, 根据线性强化假设, 此时断面的应力分布方 程为

$$
\sigma= \begin{cases}\sigma_{\mathrm{s}}+\frac{\mu \sigma_{\mathrm{s}}}{c}(z-c) & c \leqslant z \leqslant d+\frac{h}{2} \\ \sigma_{\mathrm{s}} \frac{z}{c} & d-\frac{h}{2} \leqslant z<c\end{cases}
$$

根据单侧塑性阶段的力矩平衡关系和轴向力平 衡关系可知

$$
\begin{gathered}
M=\frac{\sigma_{s} b}{6 c}\left(\frac{h}{2}+c-d\right)^{2}(h-c+d)+ \\
\frac{\mu \sigma_{s} b}{6 c}\left(\frac{h}{2}-c+d\right)^{2}(h+c-d) \\
T=\frac{\sigma_{s} b}{2 c}\left[c^{2}-\left(d-\frac{h}{2}\right)^{2}\right]+\sigma_{s} b\left(\frac{h}{2}+d-c\right)+ \\
\frac{\mu \sigma_{s} b}{2 c}\left(\frac{h}{2}+d-c\right)^{2}
\end{gathered}
$$

将式(3) (5)代入式(20)、(21)并化简可得到单 侧塑性弯曲阶段的静力平衡方程组

$$
\left\{\begin{array}{c}
t=1-\frac{1}{4 \gamma}(1+\gamma-\eta)^{2}+\frac{\mu}{4 \gamma}(1-\gamma+\eta)^{2} \\
m=\frac{1}{4 \gamma}(1+\gamma-\eta)^{2}(2-\gamma+\eta)+ \\
\frac{\mu}{4 \gamma}(1-\gamma+\eta)^{2}(2+\gamma-\eta)
\end{array}\right.
$$

根据单侧塑性阶段的应力分布方程式(19)可 知, 单侧塑性阶段下表面的应力方程为

$$
\sigma=\sigma_{\mathrm{s}} \frac{z}{c}=\sigma_{\mathrm{s}} \frac{d-\frac{h}{2}}{c}=-\sigma_{\mathrm{s}} \frac{1-\eta}{\gamma}
$$

当下表面应力达到弹性极限 $\sigma_{\mathrm{s}}$ 时, 带钢由拉伸
弯曲的单侧塑性阶段进入双侧塑性阶段, 此时的边 界条件称为第二边界条件。根据式(23)可知, 第二 边界条件为

$$
\gamma+\eta=1
$$

\subsection{2 双侧塑性弯曲阶段}

双侧塑性弯曲阶段时断面的应力分布如图 3c 所示, 根据线性强化假设, 此时断面的应力分布方 程为

$$
\sigma= \begin{cases}\sigma_{\mathrm{s}}+\frac{2 \mu \sigma_{\mathrm{s}}}{c}(z-c) & c<z \leqslant d+\frac{h}{2} \\ \sigma_{\mathrm{s}} \frac{z}{c} & -c \leqslant z \leqslant c \\ -\sigma_{\mathrm{s}}+\frac{2 \mu \sigma_{\mathrm{s}}}{c}(z+c) & d-\frac{h}{2} \leqslant z<-c\end{cases}
$$

根据双侧塑性阶段的力矩平衡关系和轴向力平 衡关系可知

$$
\begin{gathered}
M=\sigma_{\mathrm{s}} b\left[\left(\frac{h}{2}\right)^{2}-d^{2}-\frac{c^{2}}{3}\right]- \\
\frac{\mu \sigma_{\mathrm{s}} b d}{c}\left[\left(\frac{h}{2}\right)^{2}-c^{2}-\frac{d^{2}}{3}\right] \\
T=2 \sigma_{\mathrm{s}} b d\left[1+\frac{\mu}{2 c}(h-2 c)\right]
\end{gathered}
$$

将式(3) (5)代入式(26)、(27)并化简可得到双 侧塑性弯曲阶段的静力平衡方程组

$$
\left\{\begin{array}{l}
t=\eta\left[1+\frac{\mu}{\gamma}(1-\gamma)\right] \\
m=\frac{3}{2}\left(1-\eta^{2}-\frac{\gamma^{2}}{3}\right)+\frac{3 \mu \eta}{2 \gamma}\left[1-\gamma^{2}-\frac{\eta^{2}}{3}\right]
\end{array}\right.
$$

静力平衡方程组式(22)、(28)是以 $m 、 t 、 \mu$ 为参 数表示的变参数非线性方程组, 其中线性强化系数 $\mu$ 与材料属性有关。不同于理想弹塑性体假设 $(\mu=0)$ 时的线性静力平衡方程组 ${ }^{[23]}$, 本文中的变参数非线 性静力平衡方程组, 无法通过直接求解的方法得到 $\gamma 、 \eta$ 关于 $m 、 t 、 \mu$ 的表达式。

为了研究带钢在拉弯矫直过程中曲率、伸长率 随张力-弯矩的变化关系, 本文采用数值解法, 将不 同条件下 $m 、 t 、 \mu$ 的值分别代入静力平衡方程组(22) 和(28), 通过 Matlab 的嵌套 For 循环, 输出不同条 件下的 $\gamma 、 \eta$ 值, 并代入方程(12)和方程(13), 即可 得到带钢在拉弯矫直过程的塑性阶段中不同张力弯矩组合条件下, 中心层的相对曲率和上表面的伸 长率。

\section{4 不同目标函数下张力与弯矩的关系}

上述讨论中, 是以相对张力 $t$ 和相对弯矩 $m$ 为 
输入量, 以中心层相对曲率、上表面伸长率为输出 量, 通过弹塑性力学分析的方法讨论了相对张力、 相对弯矩对中心层相对曲率和上表面伸长率的影 响。但是在实际工业生产中, 通常是以中心层相对 曲率和上表面伸长率作为设定的目标值, 通过相对 张力 $t$ 和相对弯矩 $m$ 的调节来实现对中心层相对曲 率、上表面伸长率的控制。

此时中心层相对曲率、上表面伸长率成为了模 型的输入量, 相对张力 $t$ 和相对弯矩 $m$ 则转变为输 出量。将中心层相对曲率方程(12)、上表面伸长率 方程(13)分别与静力平衡方程组(22)、(28)联立, 以 $\gamma 、 \eta$ 为中间变量, 通过数值解法可求解出不同目标 值函数下相对张力与相对弯矩关系

$$
\begin{aligned}
& k_{\mathrm{c}}=G(m, t) \\
& \varepsilon_{\mathrm{a}}=F(m, t)
\end{aligned}
$$

式中, $k_{\mathrm{c}} 、 \varepsilon_{\mathrm{a}}$ 分别代表实际生产中的中心层的目标 相对曲率、上表面的目标伸长率。

\section{2 算例分析}

基于以上的计算, 以某热轧电工钢板为例进行 分析。热轧电工钢板的厚度为 $2.8 \mathrm{~mm}$, 沿着轧制方 向取样制作标准拉伸试样, 通过单向拉伸试验得到 带钢的材料参数如表 1 所示。若假设带钢为线性强 化模型, 则其线性强化系数为 $\mu=0.00237$ 。

\section{表 1 带钢材料参数}

\begin{tabular}{cccc}
\hline 屈服强度/MPa & 弹性模量/GPa & 抗拉强度/MPa & 伸长率(\%) \\
\hline 353.42 & 192.35 & 486.36 & 29.36 \\
\hline
\end{tabular}

将 $\mu=0.00237$ 分别代入静力平衡方程组(22) 和(28), 通过数值计算的方法, 可分别求得不同张 力-弯矩组合条件下 $\gamma 、 \eta$ 的值, 并代入塑性阶段的 中心层相对曲率方程(12)和上表面伸长率方程(13), 即可得到不同张力-弯矩组合条件下的中心层相对 曲率和上表面的伸长率。

\section{1 不同弯矩下曲率和伸长率随张力的变化关系}

不同相对弯矩下, 中心层相对曲率随相对张力 $t$ 的变化曲线如图 4 所示, 图 4 中从左至右相对弯矩 $m$ 逐渐减小。当相对弯矩较大时, 带钢具有较大的 初始曲率, 此时配合较小的相对张力即能满足拉矫 机对中心层相对曲率的控制要求。随着相对弯矩的 减小, 带钢的初始曲率逐渐减小, 为了获得大弯矩 条件时的同等曲率则需要较大的相对张力。此时中 心层相对曲率随相对张力的变化曲线, 会随着相对 张力的增大而进入斜率较大的区域, 此时相对张力
的细微改变会造成中心层的相对曲率出现大范围的 变化。不同相对弯矩下, 上表面伸长率随相对张力 $t$ 的变化曲线如图 5 所示, 图 5 中从左至右相对弯矩 $m$ 逐渐减小, 大张力-小弯矩条件下的张力细微的改 变, 也会造成上表面伸长率出现大范围的变化。在 实际生产过程中, 张力辊打滑等因素会导致张力在 设定值附近波动 ${ }^{[19]}$, 因此大张力-小弯矩的设定会增 加断带风险。上述分析表明, 为了保证生产稳定性, 减少断带的风险, 相对张力的设定值不应过大。

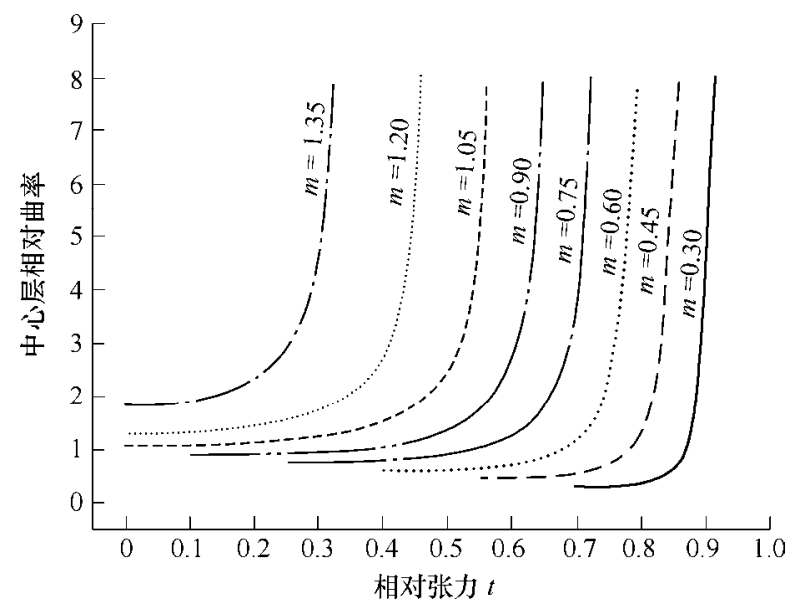

图 4 不同相对弯矩下相对曲率随相对张力的变化关系

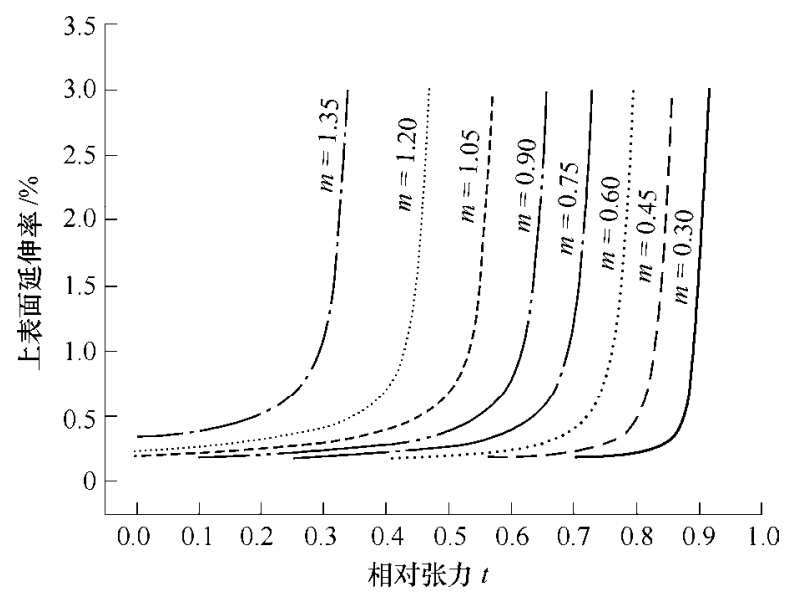

图 5 不同相对弯矩下伸长率随相对张力的变化关系

\section{2 不同张力下曲率和伸长率随弯矩的变化关系}

不同相对张力下, 中心层相对曲率随相对弯矩 $m$ 的变化曲线如图 6 所示, 图 6 中从左至右相对张 力 $t$ 逐渐减小。当相对张力较大时, 初始曲率较小; 相对张力较小时, 初始曲率较大。在大张力条件下, 中心层相对曲率随相对弯矩变化曲线很快就会进入 斜率较大的区域, 此时相对弯矩的细微改变也会造 成曲率出现大范围的变化, 从而降低生产稳定性。 不同相对张力下, 上表面伸长率随相对弯矩 $m$ 的变 化曲线如图 7 所示, 图 7 中从左至右相对张力 $t$ 逐 渐减小。在大张力条件下, 上表面伸长率随相对弯 矩的变化与图 6 中曲率随相对弯矩的变化类似。因 
此大张力的条件不利于维持生产稳定性。

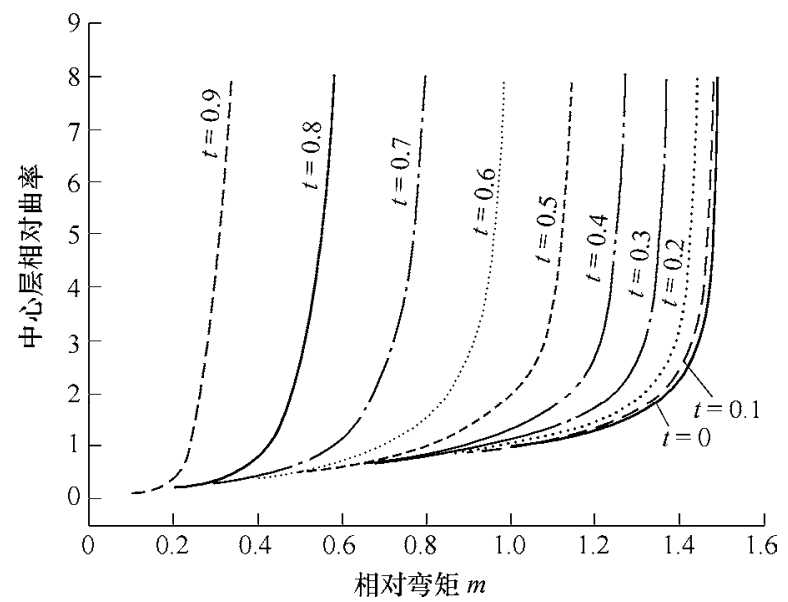

图 6 不同相对张力下相对曲率随相对弯矩的变化关系

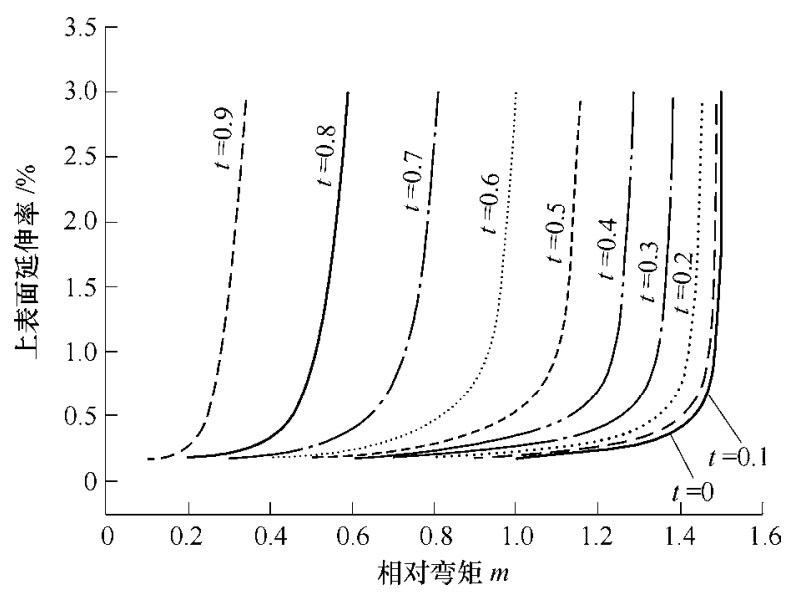

图 7 不同相对张力下伸长率随相对弯矩的变化关系

当 $t=0.1$ 时, 中心层相对曲率、上表面伸长率 随着相对弯矩的变化曲线几乎与 $t=0$ 时的曲线重 合, 此时继续减少相对张力对中心层相对曲率、上 表面伸长率的改善不明显。所以在拉弯矫直过程中, 相对张力 $t$ 的最小值通常不小于 0.1 。

\section{3 不同目标函数对张力-弯矩关系曲线的影响}

式(29)、(30)表示了不同目标曲率、伸长率下相 对张力和相对弯矩的关系。随着 $k_{\mathrm{c}} 、 \varepsilon_{\mathrm{a}}$ 目标值的不 同, 可求解出不同目标值下相对张力与相对弯矩的 关系曲线。不同目标曲率下相对张力与相对弯矩的 关系曲线如图 8 所示。由图 8 可知, 大张力-小弯矩 和小张力-大弯矩的配置方式, 均能实现对目标曲率 的控制要求。随着目标曲率的增大, 相对张力和相 对弯矩逐渐增大, 而相对张力、相对弯矩的增幅却 逐渐降低。特别的, 大张力-小弯矩条件下, 相对张 力和相对弯矩不会随着目标曲率的增大而大幅增 长。这是因为在大张力条件下 (图 4、6), 曲率对相 对张力和相对弯矩的变化较为敏感, 较小的相对张 力和相对弯矩的改变就会引起相对曲率的大幅变 化。不同目标伸长率下相对张力与相对弯矩的关系
曲线如图 9 所示, 与图 8 中以曲率为目标值的曲线 类似, 大张力-小弯矩条件下, 相对张力和相对弯矩 随着目标伸长率的变化也不是特别显著。

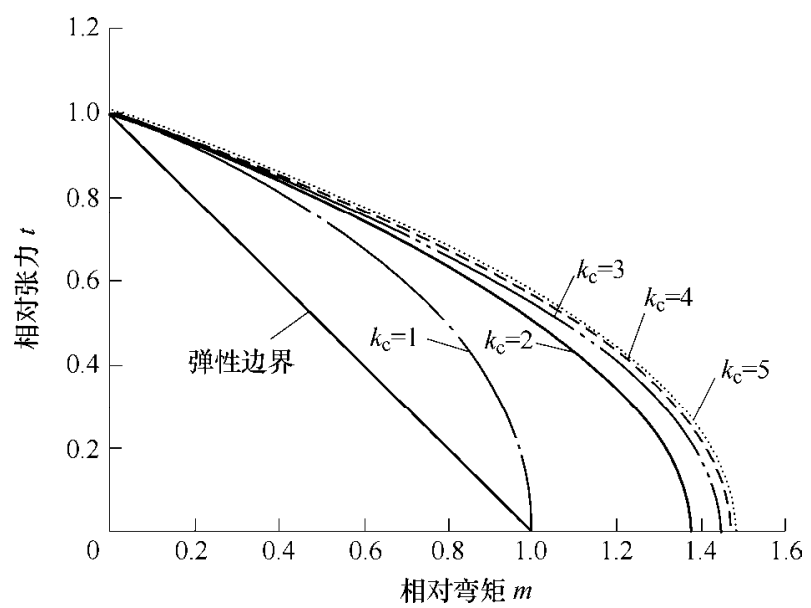

图 8 不同目标曲率下相对张力与相对弯矩的关系曲线

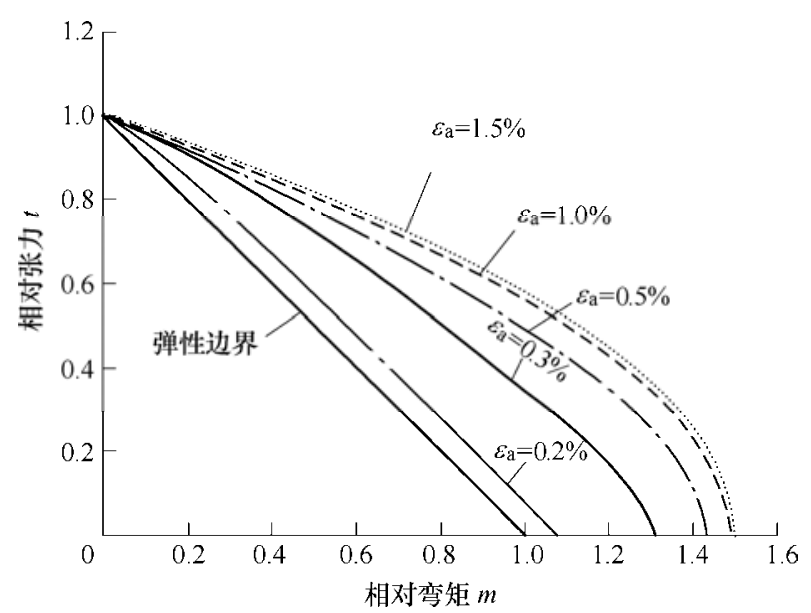

图 9 不同目标伸长率下相对张力与相对弯矩的关系曲线

上述分析表明, 虽然大张力-小弯矩和小张力大弯矩的组合条件均能满足拉弯矫直机对目标曲 率、伸长率的控制要求, 但由于大张力-小弯矩条 件下相对张力、相对弯矩的细微改变会造成相对曲 率、伸长率的大幅变化, 不利于维持生产稳定性。 为了降低中心层相对曲率和上表面伸长率对相对 张力、相对弯矩波动的敏感度, 保证生产稳定性, 拉弯矫直过程中的相对张力 $t$ 的最大值通常不大于 0.3 。

综上所述, 在工业生产中, 拉弯矫直机相对张 力的合理设定范围为 $0.1 \leqslant t \leqslant 0.3$ 。

\section{3 伸长率、曲率对酸洗时间的影响}

带钢的酸洗时间随着表面氧化铁皮剥落量的增 加而缩短。文献[18-19]的研究表明, 在拉弯矫直过 程中, 氧化铁皮的剥落量随着上表面伸长率和中心 层弯曲曲率的增加而出现饱和现象。相对于拉矫过 
程中氧化铁皮的剥落量, 酸洗时间能更直观地反映 破鳞效果, 更准确地确定拉伸、弯曲过程中的饱和 点。为了研究拉弯矫直过程中, 上表面伸长率和中 心层弯曲曲率对酸洗时间的影响, 以表 1 所示的热 轧电工钢板为研究对象, 通过电化学酸洗试验, 研 究酸洗时间随伸长率、弯曲曲率的变化关系, 建立 酸洗时间缩短率预测模型并计算酸洗饱和点。

\section{1 试验设计及方法}

在试验室条件下, 通过电子万能试验机只能进 行无张力条件下的纯弯曲试验。假设带钢为线性强 化模型, 各向同性, 忽略包辛格效应, 当拉弯矫直 模型中的相对张力 $t=0$ 时, 即可得到带钢在纯弯曲 过程中上表面伸长率、中心层相对曲率与相对弯矩 的关系式

$$
\begin{gathered}
\varepsilon_{\mathrm{a}}=\left\{\begin{array}{ll}
m \varepsilon_{\mathrm{s}} & m \leqslant 1, \\
\frac{\varepsilon_{\mathrm{s}}}{\gamma} & m \geqslant 1,
\end{array}\right. \text { 塑性阶阶段 } \\
k_{\mathrm{c}}=\left\{\begin{array}{ll}
m & m \leqslant 1, \\
\frac{1}{\gamma} & m \geqslant 1,
\end{array}\right. \text { 弹性阶段 }
\end{gathered}
$$

式中, $\gamma$ 为纯弯曲塑性阶段的弹性区的相对高度, $\gamma$ 与相对弯矩 $m$ 有如下关系

$$
2 m=3-\gamma^{2}+\frac{\mu}{\gamma}\left(2-3 \gamma+\gamma^{3}\right)
$$

纯弯曲时上表面伸长率、中心层相对曲率随 相对弯矩的变化关系如图 10 所示。当上表面伸长 率超过 $0.5 \%$ 后, 上表面伸长率随相对弯矩的变化 曲线变得较为陡峭, 此时上表面伸长率随相对弯 矩的变化较为敏感, 通过相对弯矩来调节上表面 伸长率的控制精度较低。因此纯弯曲试验不能精 确的模拟拉弯矫直过程中不同上表面伸长率的工 况。所以在试验室条件下, 采用控制精度更高的 单向拉伸试验模拟不同伸长率的工况, 拉伸试验 参数如表 2 所示。

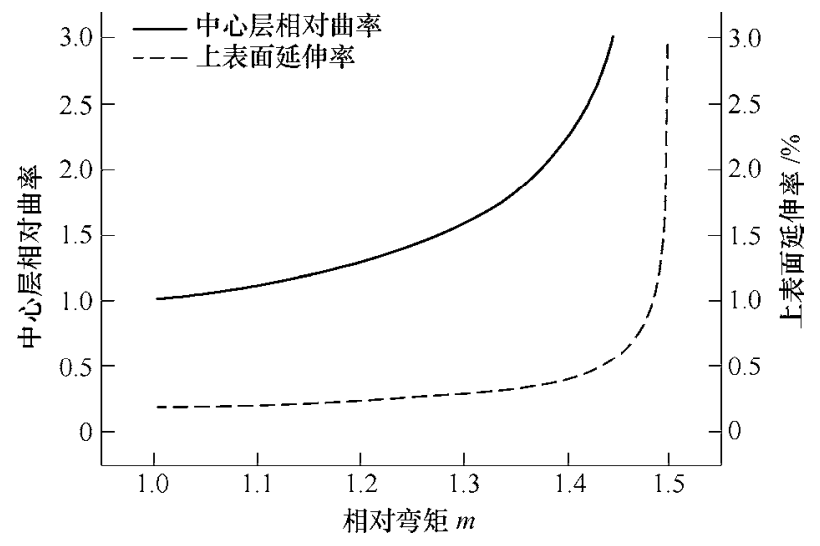

图 10 纯弯曲时伸长率、曲率随弯矩的变化关系
表 2 拉伸试验参数

\begin{tabular}{cccccccccc}
\hline 试样编号 & 1 & 2 & 3 & 4 & 5 & 6 & 7 & 8 & 9 \\
\hline 伸长率(\%) & 0 & 0.25 & 0.50 & 0.75 & 1.00 & 1.25 & 1.50 & 1.75 & 2.00 \\
\hline
\end{tabular}

而图 10 中纯弯曲时, 中心层相对曲率随相对弯 矩的变化曲线较为平缓, 通过相对弯矩调节中心层 相对曲率时能获得较好的控制精度。因此在试验室 条件下, 可通过纯弯曲试验模拟不同的中心层相对 曲率的工况。

标准的三点弯曲试验是以弯曲载荷或者压下量 作为试验控制参数。设三点弯曲试验中的载荷为 $F$, 跨距为 $L_{0}$, 根据相对弯矩的定义可知

$$
m=\frac{M}{M_{\mathrm{e}}}=\frac{F L_{0}}{4 M_{\mathrm{e}}}
$$

以相对弯矩 $m$ 为中间变量, 通过式(32)、(34) 可求出纯弯曲过程中, 中心层相对曲率与弯曲载荷 的关系。当三点弯曲试验的跨距 $L_{0}=100 \mathrm{~mm}$ 时, 可 通过控制弯曲载荷的方法实现不同中心层相对曲率 的工况, 弯曲试验参数如表 3 所示。

表 3 三点弯曲的试验参数

\begin{tabular}{cccccccccc}
\hline 试样编号 & 1 & 2 & 3 & 4 & 5 & 6 & 7 & 8 & 9 \\
\hline 相对曲率 & 0 & 0.4 & 0.8 & 1.2 & 1.6 & 2.0 & 2.4 & 2.8 & 3.2 \\
载荷 $/ \mathrm{N}$ & 0 & 74 & 148 & 212 & 241 & 254 & 261 & 265 & 269 \\
\hline
\end{tabular}

在拉伸、弯曲试验前, 先用砂纸磨去带钢一侧 的氧化铁皮, 留下单侧氧化铁皮作为研究对象。特 别的, 在进行弯曲试验时, 将带钢有氧化铁皮的一 侧朝上放置, 使得氧化铁皮能在弯曲过程中处于压 应力作用下而产生塑性变形。

试样经过拉伸、弯曲预处理后, 采用扫面电子显 微镜(SEM)对表面氧化铁皮的剥落情况进行微观检 测, 并通过拉曼光谱分析剥落的氧化铁皮成分。随后 在试样无氧化铁皮的一侧焊上导线, 并将其和与之相 邻的四个面都用环氧树脂密封。配制浓度为 $215 \mathrm{~g} / \mathrm{L}$ 的 $\mathrm{HC} 1$ 溶液作为电解液, 以 $\mathrm{AgCl}$ 作为参比电极, 以 酸洗试样作为工作电极。将 $\mathrm{HCl}$ 溶液通过水浴锅加热 至 $75{ }^{\circ} \mathrm{C}$, 待温度稳定后将电极插入 $\mathrm{HCl}$ 溶液进行电 化学酸洗。此时两电极之间由于原电池效应会产生电 势差, 且电势差随酸洗时间的增加而趋于稳定。两极 间电势差达到稳态所需的时间即为带钢的酸洗时间。

\section{2 试验结果与分析}

氧化铁皮的微观形貌随伸长率的变化如图 11 所示。由于氧化铁皮在常温下低屈服、高脆性的力 学特性 ${ }^{[24]}$, 在拉伸作用下氧化铁皮沿着试样厚度方 向产生裂纹。随着伸长率的增加, 氧化铁皮表面的 裂纹数量逐渐增多、裂纹的深度和宽度逐渐增大。 
带钢和氧化铁皮之间的剪应力随伸长率的增加而增 大。当伸长率增加至 $2 \%$ 时, 剪切力超过氧化铁皮-
基体间的结合力，部分氧化铁皮在剪应力的作用下 出现剥落。

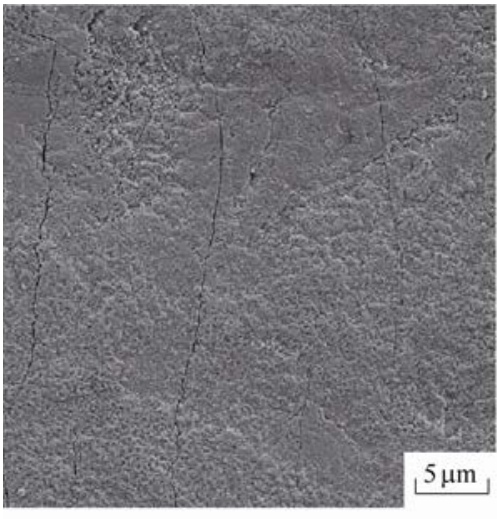

(a) 延伸率 $\varepsilon_{\mathrm{a}}=0 \%$

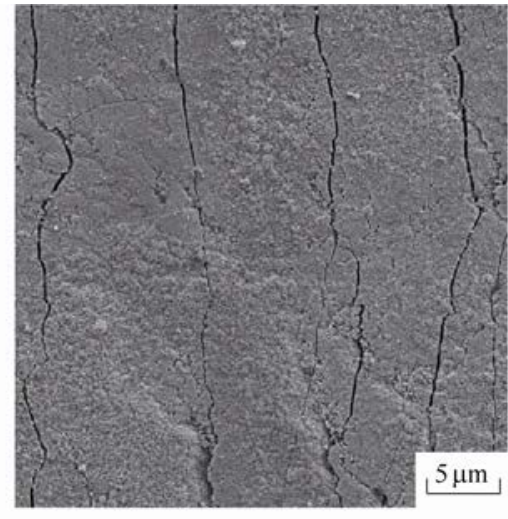

(b) 延伸率 $\varepsilon_{\mathrm{a}}=1.5 \%$

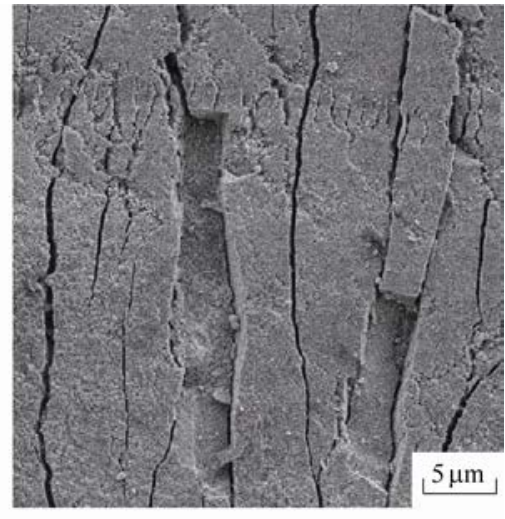

(c) 延伸率 $\varepsilon_{\mathrm{a}}=2.0 \%$

图 11 拉伸作用下氧化铁皮的微观形貌随伸长率的变化

氧化铁皮的微观形貌随相对曲率的变化如图 12 所示。在弯曲作用下, 带钢的中心层曲率逐渐 增大，氧化铁皮受到沿轧制方向的压应力作用。
脆性的氧化铁皮表面出现屈曲(图 12b), 部分氧化 铁皮的在压应力的作用下破裂并剥离带钢表面 (图 12c)。

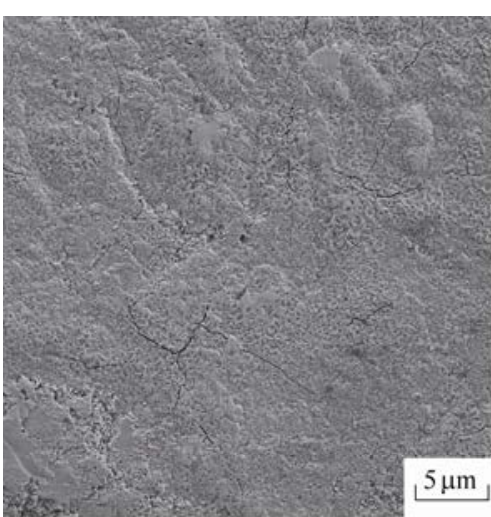

(a) 相对曲率 $k_{\mathrm{c}}=0$

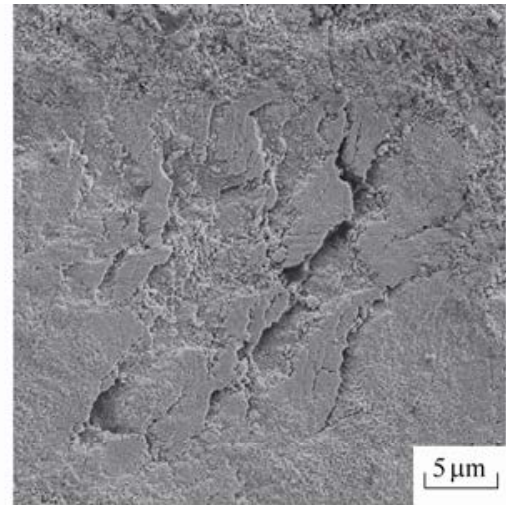

(b) 相对曲率 $k_{\mathrm{c}}=2.0$

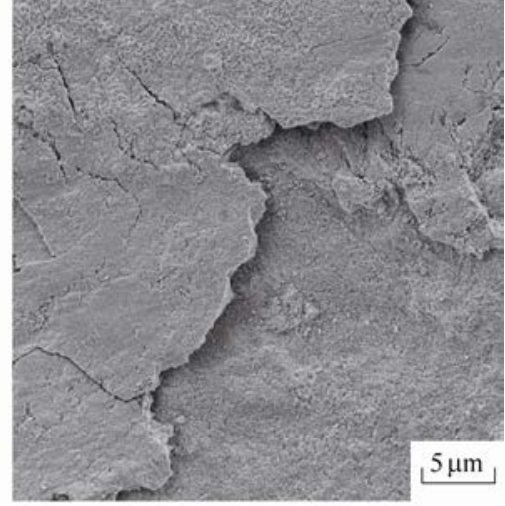

(c) 相对曲率 $k_{\mathrm{c}}=3.2$

图 12 弯曲作用下氧化铁皮的微观形貌随相对曲率的变化

伸长率达到 $2 \%$ 时氧化铁皮的微观形貌和拉曼 光谱分析结果如图 13 所示, 在拉伸作用下, 只有外 层的高价氧化物 $\mathrm{Fe}_{2} \mathrm{O}_{3}$ 会剥离带钢表面, 而内层的
$\mathrm{Fe}_{3} \mathrm{O}_{4}$ 依然完整依附于带钢基体。相对曲率达到 3.2 时氧化铁皮的微观形貌和拉曼光谱分析结果如图 14 所示, 在弯曲作用下, 也只有外层的 $\mathrm{Fe}_{2} \mathrm{O}_{3}$ 会剥

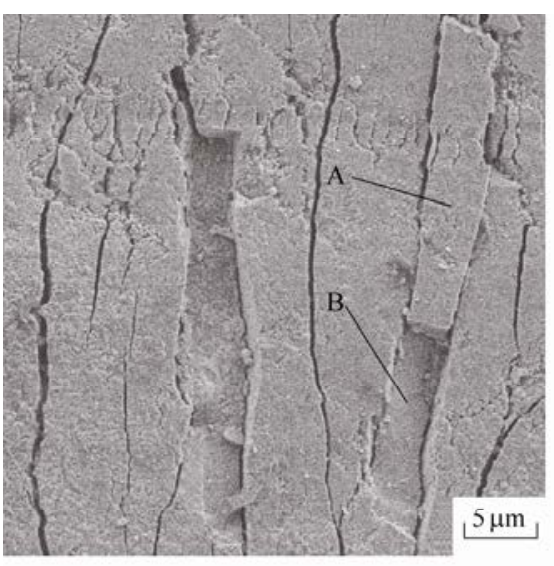

(a) 微观形貌

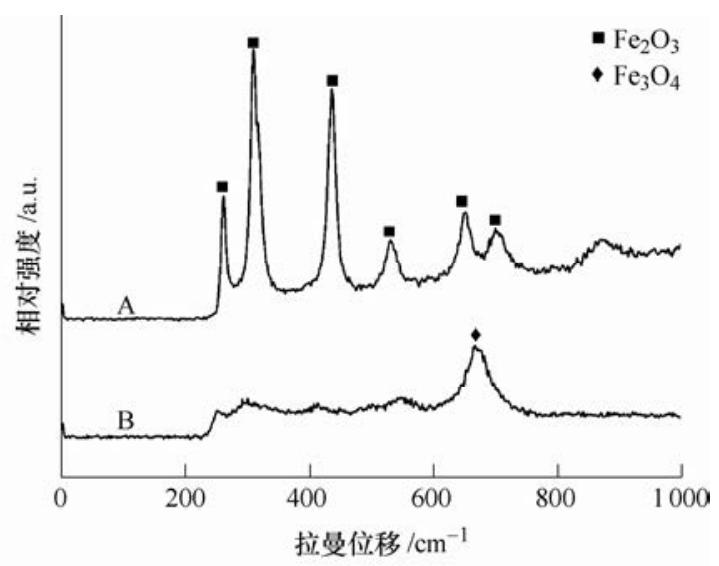

(b) 拉曼光谱

图 13 上表面伸长率 $\varepsilon_{\mathrm{a}}=2.0 \%$ 时的氧化铁皮微观形貌和拉曼光谱图 


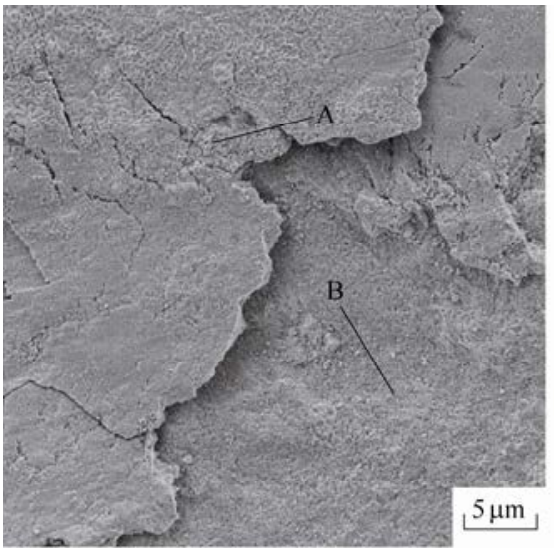

(a) 微观形貌

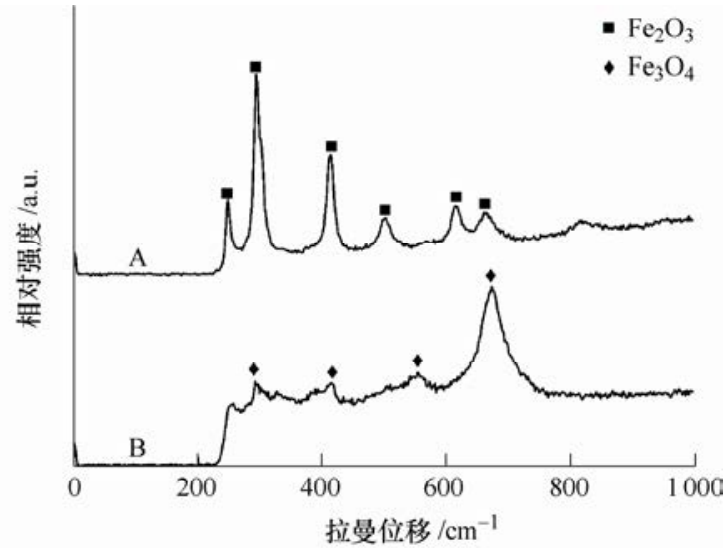

(b) 拉曼光谱

图 14 相对曲率 $k_{\mathrm{c}}=3.2$ 时的氧化铁皮微观形貌和拉曼光谱图

离带钢表面, 但是此时外层 $\mathrm{Fe}_{2} \mathrm{O}_{3}$ 的剥落面积远大 于拉伸作用时 $\mathrm{Fe}_{2} \mathrm{O}_{3}$ 的剥落面积。YAMAGUCHI 等 ${ }^{[25]}$ 研究发现, 铁的氧化物和 $\mathrm{HCl}$ 的反应速率与氧 化物中铁的化合价有关。铁的化合价越高, 其氧化 物与 $\mathrm{HCl}$ 的反应速率越慢。即在酸洗反应中, 铁的 氧化物反应速率的大小为 $\mathrm{Fe}_{2} \mathrm{O}_{3}<\mathrm{Fe}_{3} \mathrm{O}_{4}<\mathrm{FeO}$ 。随 着伸长率、弯曲曲率的增加, 外层 $\mathrm{Fe}_{2} \mathrm{O}_{3}$ 的剥落 量逐渐增加, 酸洗速率逐渐加快, 导致酸洗时间 逐渐缩短。

图 15 为通过电化学酸洗试验得到的带钢酸洗 时间随伸长率、相对曲率的变化曲线。由图 15 可知, 带钢未经拉伸、弯曲处理时的酸洗时间为 $30.4 \mathrm{~s}$ 。 随着伸长率和相对曲率的增加, 带钢的酸洗时间逐 渐降低并趋于稳定。在拉伸作用下, 当伸长率增加 至 $2 \%$ 时, 带钢的酸洗时间为 $17.1 \mathrm{~s}$; 在弯曲作用下, 当相对曲率的增加至 3.2 时, 带钢的酸洗时间为 16.2 S。由图 15 可知, 相对于伸长率的增加, 相对曲率 的增加能更显著的缩短酸洗时间。

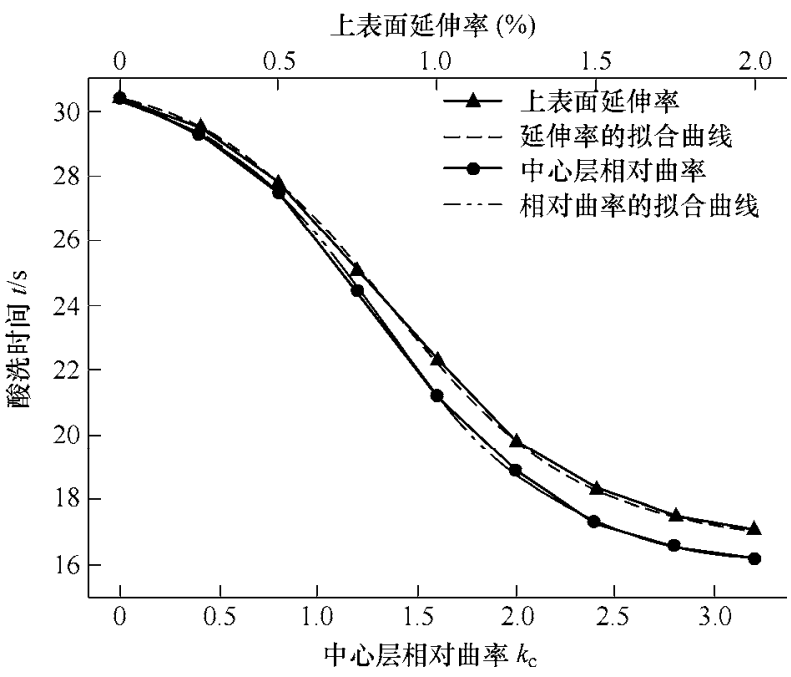

图 15 酸洗时间随伸长率、相对曲率的变化曲线

在拉伸作用下，氧化铁皮表面会产生沿厚度方
向的裂缝, 并伴随着少量 $\mathrm{Fe}_{2} \mathrm{O}_{3}$ 的剥离。其酸洗时 间的缩短, 主要是依靠酸洗时 $\mathrm{HCl}$ 溶液渗入氧化铁 皮表面的裂缝, 与内层易溶解的低价氧化物反应而 实现。随着氧化反应的进行, 内层的低价氧化物在 $\mathrm{HCl}$ 溶液作用下发生横向溶解, 使得氧化铁皮发生 剥离。氧化铁皮表面的裂缝数量、宽度、深度随着 伸长率的增大而增多(图 11), 通过裂缝渗入到基体 的 $\mathrm{HCl}$ 溶液逐渐增多, 酸洗时间随之缩短。当伸长 率的足够大时, 内层氧化物与 $\mathrm{HCl}$ 的反应达到饱和, 此时酸洗时间不再随着伸长率的增加而缩短。而在 弯曲作用下, 带钢的外层 $\mathrm{Fe}_{2} \mathrm{O}_{3}$ 在屈曲作用系出现 大面积剥落, 使得 $\mathrm{HCl}$ 溶液能直接跟裸露的低价氧 化物发生反应, 加速酸洗进程。随着弯曲程度增大, 氧化铁皮的剥落面积达到饱和, 酸洗时间随相对曲 率的变化曲线趋于平稳。由于弯曲时屈曲作用能直 接剥离掉大量的外层 $\mathrm{Fe}_{2} \mathrm{O}_{3}$, 因此弯曲作用能更显 著的缩短带钢的酸洗时间。

\section{3 酸洗模型}

由图 15 可知, 酸洗时间与伸长率、相对曲率的 关系呈 S 形变化, 且有三段较为明显的区间: (1) 当 伸长率、相对曲率较小时, 酸洗时间的变化速率较 慢, 此阶段为潜伏期; (2) 随着伸长率、相对曲率的 逐渐增大, 酸洗时间迅速缩短, 此阶段为加速期;

(3) 当伸长率、相对曲率增加至一定程度时, 酸洗时 间的变化速率又逐渐降低, 直至趋于稳定, 此阶段 为稳定期。

已有学者 ${ }^{[26-27]}$ 采用多种数学模型对这种 S 型曲 线进行拟合分析后发现通过 Boltzmann 函数模型拟 合后决定系数 $R^{2}$ 能达到 0.99 以上。故本文采用 Boltzmann 函数模型对酸洗时间的散点图进行拟合 分析, 其函数如式(35)所示

$$
Y=A_{2}+\frac{A_{1}-A_{2}}{1+\exp \left[\left(x-x_{0}\right) / p\right]}
$$


式中, $Y$ 为酸洗时间, $x$ 为自变量表示伸长率、相对 曲率的变化, $A_{1} 、 A_{2} 、 x_{0} 、 p$ 、为模型参数。其中 $\left.Y\right|_{x \rightarrow-\infty}=\left.A_{1} 、 Y\right|_{x \rightarrow \infty}=A_{2}$ 为 $S$ 型曲线的两条渐近线, $A_{1}$ 表示带钢未经拉伸、弯曲处理时的酸洗时间, $A_{2}$ 表示当伸长率、相对曲率趋于无穷大时的酸洗时间。 $x_{0}$ 为酸洗时间变化的拐点, 表示当酸洗时间缩短 $50 \%$ 时, 伸长率、相对曲率的大小, $\mathrm{S}$ 型曲线关于 点 $\left[x_{0},\left(A_{1}+A_{2}\right) / 2\right]$ 对称。当 $x=x_{0}$ 时酸洗时间的变化 速率最快, 此时 $\mathrm{S}$ 型曲线的斜率即为 $p$ 。

表 4、5 为对两条 $\mathrm{S}$ 形曲线拟合后的拟合参数。 两组模型拟合后的决定系数分别为 0.99992 和 0.99976 , 各参数的标准差中最大的仅为 0.148 , 说 明模型均具有较好的拟合精度。

表 4 酸洗时间随伸长率变化模型的拟合参数

\begin{tabular}{ccccc}
\hline 模型参数 & $A_{1}$ & $A_{2}$ & $x_{0}$ & $p$ \\
\hline 拟合值 & 31.25 & 16.79 & 0.85 & 0.30 \\
标准差 & 0.081 & 0.052 & 0.005 & 0.005 \\
\hline
\end{tabular}

表 5 酸洗时间随相对曲率变化模型的拟合参数

\begin{tabular}{ccccc}
\hline 模型参数 & $A_{1}$ & $A_{2}$ & $x_{0}$ & $p$ \\
\hline 拟合值 & 31.31 & 15.98 & 1.31 & 0.46 \\
标准差 & 0.148 & 0.088 & 0.013 & 0.013 \\
\hline
\end{tabular}

根据表 4、5 中伸长率、相对曲率的拟合参数, 以及式(35)所示的酸洗时间预测模型, 可分别构建 酸洗时间随伸长率变化的预测模型 $Y_{\varepsilon}$, 以及酸洗时 间随相对曲率变化的预测模型 $Y_{k}$

$$
\begin{aligned}
& Y_{\varepsilon}=16.79+\frac{14.46}{1+\exp \left[\left(x-x_{0}\right) / 0.30\right]} \\
& Y_{k}=15.98+\frac{15.33}{1+\exp \left[\left(x-x_{0}\right) / 0.46\right]}
\end{aligned}
$$

设 $\Delta Y$ 为酸洗时间随伸长率、相对曲率变化的 缩短量

$$
\Delta Y=A_{1}-Y=A_{1}-A_{2}-\frac{A_{1}-A_{2}}{1+\exp \left[\left(x-x_{0}\right) / p\right]}
$$

由 Boltzmann 函数模型可知, 当 $x$ 趋近于 $\infty$ 时, $Y=A_{2}$, 此时酸洗时间的最大缩短量为

$$
\Delta Y_{\max }=A_{1}-A_{2}
$$

设 $q$ 为酸洗时间随伸长率、曲率变化的缩短率

$$
\begin{gathered}
q=\frac{\Delta Y}{\Delta Y_{\max }}=\frac{A_{1}-A_{2}-\frac{A_{1}-A_{2}}{1+\exp \left[\left(x-x_{0}\right) / p\right]}}{A_{1}-A_{2}}= \\
1-\frac{1}{1+\exp \left[\left(x-x_{0}\right) / p\right]}
\end{gathered}
$$

式(40)为酸洗时间缩短率的预测模型, 该模型
的参数只与酸洗时间变化的拐点 $x_{0}$ 和拐点处的斜 率 $p$ 有关。

以酸洗时间缩短率为目标值, 通过式(40)可得 到伸长率、相对曲率随酸洗时间缩短率的变化关系 (表 6)。由上述的分析可知, 当拉伸试验中带钢的伸 长率增加至 $0.85 \%$ 时, 酸洗时间能缩短 $50 \%$; 弯曲 试验中带钢中心层的相对曲率达到 1.31 时也能使酸 洗时间缩短 50\%。随着伸长率、相对曲率的进一步 增加, 酸洗曲线趋于水平, 酸洗开始出现饱和现象。 若以酸洗时间缩短 $90 \%$ 作为酸洗饱和点, 由表 6 可知, 当纯拉伸条件下带钢的伸长率为 $1.51 \%$ 、纯 弯曲条件下带钢的相对曲率为 2.33 时, 酸洗达到 饱和。

表 6 伸长率、相对曲率随酸洗时间减少率的变化

\begin{tabular}{cccccc}
\hline 试验组别 & $q=50 \%$ & $q=60 \%$ & $q=70 \%$ & $q=80 \%$ & $q=90 \%$ \\
\hline 伸长率(\%) & 0.85 & 0.97 & 1.10 & 1.27 & 1.51 \\
相对曲率 & 1.31 & 1.49 & 1.69 & 1.95 & 2.33 \\
\hline
\end{tabular}

\section{4 相对张力-相对弯矩的调节域}

由图 8、图 9 可知, 当相对曲率、伸长率为唯 一确定的目标值时, 能通过式(29)、(30)绘制出当 前目标值下相对张力-相对弯矩的关系图。根据酸 洗时间缩短率预测模型的结果, 分别以酸洗饱和时 的中心层相对曲率 $k_{\mathrm{c}}=2.33$ 和上表面伸长率 $\varepsilon_{\mathrm{a}}=1.51 \%$ 作为目标值, 构建相对张力-相对弯矩的 关系曲线, 不同目标值下, 相对张力-相对弯矩的 关系曲线如图 16 所示。图中曲线 a 为中心层相对 曲率 $k_{\mathrm{c}}=2.33$ 时的相对张力-相对弯矩关系曲线, 曲 线 $\mathrm{b}$ 为上表面伸长率 $\varepsilon_{\mathrm{a}}=1.51 \%$ 时的相对张力-相对 弯矩关系曲线。

在生产过程中, 由于张力辊打滑等因素的影响, 过高的伸长率设定值会导致更多伸长率损失。同时, 过大的伸长率还会增大系统负荷、增加断带风险, 造成不必要的安全隐患 ${ }^{[19]}$ 。因此在实际生产中, 拉 弯矫直机的最大负荷伸长率一般不超过 $2 \%$ 。不同 于前文推导中提到的上表面伸长率, 此处的伸长率 指的是带钢中心层的伸长率。根据假设(3)可知, 拉 伸弯曲塑性阶段中心层的伸长率公式为

$$
\varepsilon_{\mathrm{c}}=\frac{Z}{\rho}=\frac{h}{2 \rho} \eta=\frac{h}{2} \frac{k}{\rho_{\mathrm{e}}} \eta=k \varepsilon_{\mathrm{s}} \eta=\frac{\varepsilon_{\mathrm{s}} \eta}{\gamma}
$$

将中心层伸长率方程(41)分别与静力平衡方程 组(22)、(28)联立, 以 $\gamma 、 \eta$ 为中间变量, 通过数值 解法可计算不同中心层伸长率下相对张力-相对弯 矩的关系 


$$
\varepsilon_{\mathrm{c}}=F^{\prime}(m, t)
$$

当式(42)中的 $\varepsilon_{\mathrm{c}}=2 \%$ 时, 即可构建出拉弯矫直 机处于最大伸长率负荷下相对张力-相对弯矩的关 系曲线, 如图 16 中的曲线 $c$ 所示。

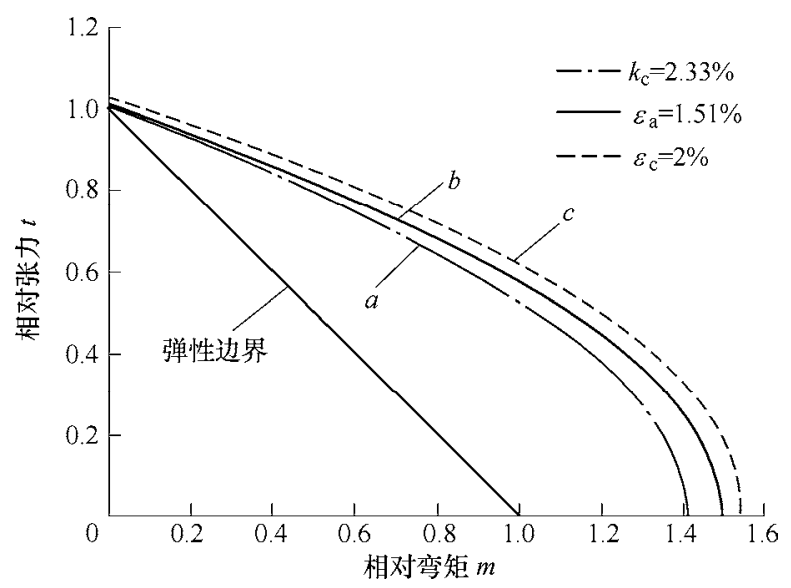

图 16 不同目标值下相对张力-相对弯矩的关系曲线

不同目标值下相对张力-相对弯矩的关系曲线 如图 16 所示。在满足酸洗饱和点要求的前提下, 以 中心层相对曲率 $k_{\mathrm{c}}=2.33$ 为目标值时的相对张力和 相对弯矩远小于以上表面伸长率 $\varepsilon_{\mathrm{a}}=1.51 \%$ 为目标时 的相对张力和相对弯矩。带钢在拉弯矫直过程中, 受拉侧和受压侧交替变化, 当相对张力、相对弯矩 大于曲线 $a 、 b$ 中的较小值时, 就能显著的提升酸洗 效率。由图 16 可知, 可将 $k_{\mathrm{c}}=2.33$ 时的相对张力相对弯矩关系曲线 $\mathrm{a}$ 设为相对张力-相对弯矩配置时 的左边界。同时为了减小拉弯矫直机的系统负荷、 降低断带风险, 中心层伸长率应小于最大负荷伸长 率。由图 16 可知, 可将 $\varepsilon_{\mathrm{c}}=2 \%$ 时的相对张力-相对 弯矩关系曲线 $\mathrm{c}$ 设为相对张力-相对弯矩配置时的右 边界。由图 4 9 可知, 小张力-大弯矩的条件既能 满足相对曲率、伸长率的控制要求, 还能提高设备 的稳定性, 其中相对张力的合理设定范围为 $0.1 \leqslant t$ $\leqslant 0.3$ 。因此通过小张力-大弯矩条件下的相对张力 临界值 $(t=0.3 、 t=0.1)$ 可分别确定相对张力-相对弯矩 配置时的上下边界。

综上所述, 带钢在拉弯矫直过程中, 合理的张 力-弯矩调节域如图 17 所示。图 17 中曲线 $A D$ 代表 以带钢达到酸洗饱和点时的中心层曲率 $\left(k_{\mathrm{c}}=2.33\right)$ 作 为目标值时的相对张力 -相对弯矩关系, 当相对张 力、相对弯矩的设定值大于曲线 $A D$ 时, 酸洗时间 能缩短 $90 \%$, 显著的提高了酸洗效率; 曲线 $B C$ 代 表带以带钢中心层的最大负荷伸长率 $\left(\varepsilon_{\mathrm{c}}=2 \%\right)$ 作为 目标值时的相对张力-相对弯矩关系, 当相对张力、 相对弯矩的设定值小于曲线 $B C$ 时, 能有效降低系 统负荷; 曲线 $A B 、 D C$ 分别代表小张力-大弯矩条件
下相对张力的上下临界值, 当相对张力值处于此区 间时, 能满足小张力-大弯矩的条件。综上所述, 当拉弯矫直机的张力 - 弯矩组合参数落在张力 - 弯 矩调节域内时, 不仅能显著提高酸洗效率, 还能减 小系统负荷、降低断带风险, 保证生产的高效性和 稳定性。

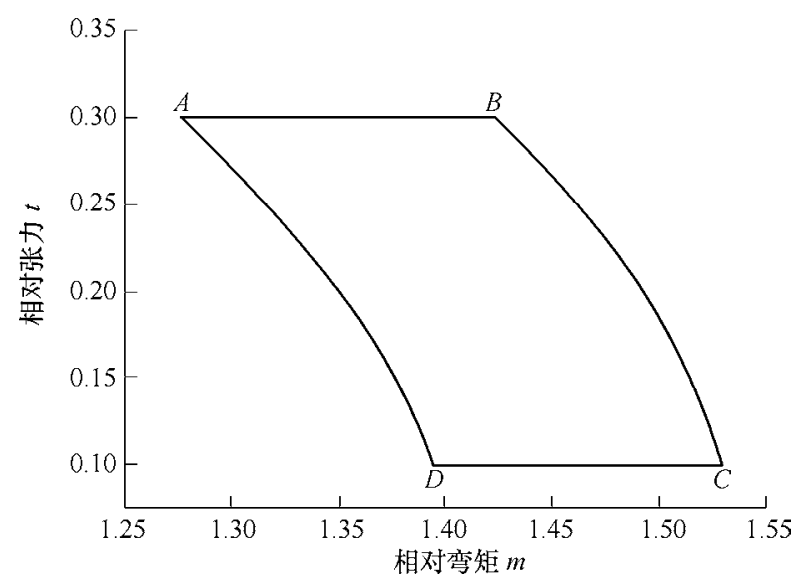

图 17 张力-弯矩的调节域

\section{5 结论}

(1) 采用弹塑性力学的分析方法, 建立了带钢 在拉弯矫直过程中曲率、伸长率随张力和弯矩变化 的数学模型。结果表明大张力-小弯矩、小张力-大 弯矩的配置方式, 均能实现对目标曲率和目标伸长 率的控制要求。由于大张力-小弯矩条件下, 张力、 弯矩的细微改变会造成曲率、伸长率的大幅变化, 不利于维持生产稳定性。因此在工业生产中, 拉弯 矫直机通常采用小张力-大弯矩的组合条件, 其中相 对张力的合理设定范围为 $0.1 \leqslant t \leqslant 0.3$ 。

(2) 通过电化学酸洗试验研究发现, 某热轧电 工钢板的酸洗时间随着曲率、伸长率的增加逐渐缩 短, 且呈 $\mathrm{S}$ 型变化。当曲率、伸长率增加至一定程 度时, 酸洗出现饱和现象, 此时酸洗时间不再随曲 率、伸长率的提高而显著缩短。通过建立酸洗时间 缩短率预测模型, 计算出某热轧电工钢板在达到酸 洗饱和点时的中心层相对曲率和上表面伸长率分别 为 2.33 和 $1.51 \%$ 。

(3) 通过理论分析与试验相结合的方法, 以弯 曲曲率、伸长率为中间变量, 分析了拉弯矫直参数 对酸洗效率的影响。以不同目标值下的张力-弯矩曲 线作为左右边界, 通过小张力-大弯矩条件下的张力 临界值确定上下边界, 计算出合理的张力-弯矩调节 域。本文的研究结果为酸洗前拉弯矫直过程参数组 合优化提供了理论依据。 


\section{参 考 文 献}

[1] 张亮, 陈兵, 付光, 等. 冷轧拉伸弯曲矫直机工艺参数 设定影响因素研究[J]. 轧钢, 2014, 31(6): 5-8.

ZHANG Liang, CHEN Bing, FU Guang, et al. Research of inflluence factors on process parameters setting of cold rolling tension leveller[J]. Steel Rolling, 2014, 31(6): 5-8.

[2] LIU Y, PENG Y. The Simulation analysis of tension leveling for improving the shape defects of strip[J]. Advanced Materials Research, 2013, 634-638(1) : 2818-2823.

[3] 王小红, 张丁非, 彭建, 等. 拉伸矫直数学模型及试验 验证[J]. 机械工程学报, 2011, 47(8): 66-70.

WANG Xiaohong, ZHANG Dingfei, PENG Jian, et al. Mathematical model of tension straightening process and its experiment validation[J]. Journal of Mechanical Engineering, 2011, 47(8): 66-70.

[4] MORRIS J W, HARDY S J, THOMAS J T. Effects of tension levelling process parameters on cold rolled strip characteristics using a designed factorial analysis approach[J]. Ironmaking \& Steelmaking, 2013, 32(5): 443-448.

[5] MATHIEU N, POTIER-FERRY M, ZAHROUNI H. Reduction of flatness defects in thin metal sheets by a pure tension leveler[J]. International Journal of Mechanical Sciences, 2017, 122: 267-276.

[6] 张清东, 周岁, 张晓峰, 等. 薄带钢拉矫机浪形矫平过 程机理建模及有限元验证[J]. 机械工程学报, 2015, 51(2): 49-57.

ZHANG Qingdong, ZHOU Sui, ZHANG Xiaofeng, et al. Analytic modeling and corroborating by fem of tension leveling process of thin buckled steel strip[J]. Journal of Mechanical Engineering, 2015, 51(2): 49-57.

[7] WANG D C, LIU H M, LIU J. Research and development trend of shape control for cold rolling strip[J]. Chinese Journal of Mechanical Engineering, 2017，30(5): 1-14.

[8] 刘现翠, 苏兰海, 李忠富, 等. 宝钢 2030 酸洗机组中 拉矫机和平整机的伸长率分配 [J]. 北京科技大学学报, 2005, 27(5): 609-612.

LIU Xiancui, SU Lanhai, LI Zhongfu, et al. Elongation assignment of steel plates between a tension leveler and a skin pass mill for the Acid-Refined 2030 in BaoSteel[J]. Journal of University of Science \& Technology Beijing, 2005, 27(5): 609-612.

[9] 叶东东, 陈建钧, 王忠建. 不同应力状态下带钢的破鳞 机理[J]. 钢铁研究学报, 2016, 28(1): 64-70.

YE Dongdong, CHEN Jianjun, WANG Zhongjian. Spalling mechanisms of oxide scale of strip under different stress states[J]. Journal of Iron \& Steel Research. 2016, 28(1): 64-70.

[10] 叶东东, 邵素娟, 阴子良, 等. 拉矫参数对带钢氧化皮 剥离及酸洗性能的影响 [J]. 表面技术, 2015，44(7): 125-130.

YE Dongdong, SHAO Sujuan, YIN Ziliang, et al. Influence of descaling parameters on the spalling and pickling properties of oxide scales[J]. Surface Technology, 2015, 44(7): 125-130.

[11] 邵素娟, 叶东东, 陈建钧. 破鳞参数对带钢酸洗速率影 响规律的研究 [J]. 表面技术, 2015，44(2): 119-122.

SHAO Sujuan, YE Dongdong, CHEN Jianjun. Study of the law for the effect of descaling parameters on pickling speed[J]. Surface Technology, 2015，44(2): 119-122.

[12] 陈兵, 何名成. 冷轧带钢拉矫机破鳞参数优化与试验研 究[C]//宝钢学术年会. 上海: 中国金属学会, 2015.

CHEN Bing, HE Mingcheng. Research on parameter optimization \& experiment of descaling in tension leveler for cold rolled strips[C]//Baosteel BAC. Shanghai: The Chinese Society for Metals, 2015.

[13] 杨子良, 王欣, 郑涛, 等. 拉矫工艺参数对带钢酸洗速 率影响的试验研究[J]. 轧钢, 2013, 30(2): 28-30.

YANG Ziliang, WANG Xin, ZHENG Tao, et al. Study on effect of stretcher lever parameters on pickling time[J]. Steel Rolling, 2013, 30(2): 28-30.

[14] 付松岳, 任勇, 程晓茹, 等. 高碳钢盘条三次氧化铁皮 机械剥离性能 $[\mathrm{J}]$. 钢铁，2016，51(3): 73-77.

FU Songyue, REN Yong, CHENG Xiaoru, et al. Descaling property of third oxide scale of high carbon steel wire[J]. Iron \& Steel, 2016, 51(3): 73-77.

[15] EVANS H E， LOBB R C. Conditions for the initiation of oxide-scale cracking and spallation[J]. Corrosion Science, 1984, 24(3): 209-222.

[16] EVANS H E. Cracking and spalling of protective oxide layers[J]. Materials Science and Engineering: A, 1989, 120(1): 139-146.

[17] EVANS H E. Spallation of oxide from stainless steel AGR nuclear fuel cladding: mechanisms and consequences[J]. Materials Science and Technology, 1988, 4(5): 414-420.

[18] 王斌, 叶东东, 陈建钧, 等. 带钢氧化膜受压断裂试验 及数值分析 $[J]$. 钢铁研究学报, 2017, 29(9): 762-767. WANG Bin, YE Dongdong, CHEN Jianjun, et al. Experimental and numerical analysis of compression fracture for strip steel oxidation film[J]. Journal of Iron \& Steel Research, 2017, 29(9): 762-766.

[19] LI T, CHEN X, XU Y. Research into technology of Bao Steel's pickling tension leveler[J]. Journal of Iron and Steel Research International(China), 1997，4(1): 24-30. [20] 王尚, 杨荃, 任云鹤, 等. 热轧带钢氧化铁皮拉伸开裂 
行为 [J]. 工程科学学报, 2017, 39(10): 1540-1545.

WANG Shang, YANG Quan, REN Yunhe, et al. Tensile cracking behavior of oxide scale in hot-rolled steel[J]. Chinese Journal of Engineering, 2017, 39(10): 1540-1545.

[21] 何安瑞, 刘东冶, 刘超. 塑性强化材料辊式矫直力学行 为研究 [J]. 机械工程学报, 2016, 52(18): 85-91.

HE Anrui, LIU Dongye, LIU Chao. Research on roller straightening mechanical behavior of plastic hardening material[J]. Journal of Mechanical Engineering, 2016, 52(18): 85-91.

[22] 余同希, 章亮炽. 塑性弯曲理论及其应用[M]. 北京: 科学出版社, 1992.

YU Tongxi, ZHANG Liangchi. Plastic bending: theory and applications[M]. Beijing: Science Press, 1992.

[23] YU T X, JOHNSON W. Influence of axial force on the elastic-plastic bending and springback of a beam[J]. Journal of Mechanical Working Technology, 1982, 6(1): 5-21.

[24] SUAREZ L, HOUBAERT Y, EYNDE X V, et al. High temperature deformation of oxide scale[J]. Corrosion Science, 2009, 51(2): 309-315.

[25] YAMAGUCHI S, YOSHIDA T, SAITO T. Improvement in descaling of hot strip by hydrochloric acid[J]. ISIJ international, 1994, 34(8): 670-678.

[26] 薛宪营, 刘奉家, 秦军, 等. HSLA Q345D 中厚板韧脆 转变温度测定与分析 [J]. 钢铁, 2011, 46(10): 94-98.

XUE Xianying, LIU Fengjia, QIN Jun, et al. Determination and analysis of tough-brittle transition temperature for HSLA Q345D heavy plate[J]. Iron \& Steel, 2011, 46(10): 94-98.

[27] FEI L, XU G H, LIN L, et al. Least squares evaluations for form and profile errors of ellipse using coordinate data[J]. Chinese Journal of Mechanical Engineering, 2016, 29(5): 1020-1028.

作者简介: 张赵宁, 男, 1988 年出生, 博士研究生。主要研究方向为热 轧带钢氧化铁皮的控制。

E-mail: zznsunny@163.com

张杰, 男, 1960 年出生, 博士, 教授, 博士研究生导师。主要研究方向 为冶金机械工作行为与控制、塑性加工工艺与设备等。

E-mail: zhangjie@ustb.edu.cn

孔宁(通信作者), 男, 1984 年出生, 博士, 讲师。主要研究方向为轧制 界面润滑、高温氧化腐蚀、机械力学行为与表面工程等。

E-mail: kongning@ustb.edu.cn

李洪波, 男, 1982 年出生, 博士, 副教授, 硕士研究生导师。主要研究 方向为冶金机械力学行为与控制等。

E-mail: lihongbo@ustb.edu.cn 